\title{
Evaluation of trip and car sharing concepts for home health care services
}

\author{
Christian Fikar $^{1}$ (D) Patrick Hirsch ${ }^{1}$
}

Published online: 26 August 2016

(C) The Author(s) 2016. This article is published with open access at Springerlink.com

\begin{abstract}
Routing and scheduling of home health care services usually focuses on the case where each nurse operates a separate vehicle. With increasing urbanisation, limited availability of parking spaces and stricter environmental regulations, service providers are starting to investigate car and trip sharing concepts as potential alternatives. This paper numerically investigates a car sharing concept as well as operating a transport system, which delivers and picks up nurses to and from clients combined with the additional option of walking. Different geographic distributions are investigated to identify beneficial settings for successful implementation considering various objectives of decision makers. The evaluation shows that trip sharing performs best if long service durations exist, long delays for parking occur and in areas where clients are both geographically distributed randomly and in clusters. Additionally, facilitating walking as well as trip or car sharing reduces the number of required vehicles substantially. Nevertheless, for walking and trip sharing, travel durations are prone to increase compared to classical planning approaches.
\end{abstract}

Keywords Trip sharing - Car sharing · Home health care - Sustainability · Synchronization $\cdot$ Routing and scheduling

Financial support of the programme iv2splus, provided by the Austrian Research Promotion Agency and the Federal Ministry for Transport, Innovation and Technology is gratefully acknowledged (Project Number 835770).

Christian Fikar

christian.fikar@boku.ac.at

Patrick Hirsch

patrick.hirsch@boku.ac.at

1 Institute of Production and Logistics, University of Natural Resources and Life Sciences, Vienna, Feistmantelstraße 4, 1180 Vienna, Austria 


\section{Introduction}

Recent trends indicate a shift from long-term care provided in hospitals or nursing homes to services performed at clients' homes (Rosenfeld and Russell 2012). Whilst the majority of tasks are nursing services and counselling, demand for short-term care is particularly expected to increase (Dorin et al. 2014). As a result, home health care (HHC) providers face increased organisational efforts, whilst being additionally exposed to various risks affecting service quality as well as client and employee satisfaction (Rest et al. 2012). An overview of various operations managementrelated decisions of HHC providers is provided in Matta et al. (2012). On an operational level, in particular, routing and scheduling of nurses is complex. As a consequence, the development of routing and scheduling procedures helps HHC providers to decrease administrative efforts and enables better fulfilment of clients' requests. Solution procedures to route and schedule nurses, however, aim to solve operational tasks and are rarely used to investigate strategic or tactical questions such as fleet management or mode of transport choices. As a consequence, current practices are seldom questioned, hindering the implementation of innovative transport concepts.

Motivated by a project in cooperation with the Austrian Red Cross, a major HHC provider in Austria, this work investigates the impact of car and trip sharing in the context of HHC operations. Expanding on the trip sharing concept presented in Fikar and Hirsch (2015), we compare trip sharing to each nurse operating a separate vehicle, develop a car sharing procedure, investigate performance in different geographic distributions of clients and analyse different objectives of decision makers. Although the study focuses on HHC services, results are of further interest to other home service industries facing similar challenges. Consequently, the contribution of this paper is twofold: (1) extensively comparing the impact of trip and car sharing concepts with the current practice of each nurse operating a separate vehicle, and (2) providing decision makers with beneficial settings for implementation.

The remainder of this paper is organised as follows: Sect. 2 gives background information and reviews related literature. Section 3 describes the analysed optimisation problems, whilst Sect. 4 introduces the solution procedures and experimental design. Results of the evaluation are presented and discussed in Sect. 5 and concluding remarks in Sect. 6.

\section{Background}

Trip sharing (also referred to as ride sharing), where multiple travellers share one vehicle for their individual trips simultenously, is used in various sectors by both public and private organisations and currently highly encouraged by governmental and intergovernmental agencies world-wide (ADB 2010; EC 2011; US-DOT 2013). An extensive overview of its challenges and future potentials is provided by Furuhata et al. (2013), who list design, arrangement and trust issues as major obstacles for agencies utilising trip sharing. In contrast, car sharing refers in this 
work to settings where two or more travellers share one vehicle at various times of the day, however, not simultaneously. Most trip sharing concepts deal with the transport of customers, however, little work is found on organised trip sharing provided by employers, with the exception of commuter carpools to and from work, which have been studied widely (e.g., Wartick 1980; Teal 1987; Ferguson 1997; Vanoutrive et al. 2012). For HHC services, due to high complexity of operations, social importance and current challenges such as limited availability of parking spaces and stricter environmental regulations, numerical evaluations of trip and car sharing concepts are, as a consequence, of interest.

\subsection{Routing and scheduling of HHC services}

HHC routing and scheduling with a single mode of transport for each nurse, mostly separate cars, is predominantly considered in literature (e.g., Begur et al. 1997; Chahed et al. 2009; Trautsamwieser et al. 2011; Rasmussen et al. 2012; Cappanera and Scutellá 2015; Yalçındağ et al. 2016) and is closely related to the vehicle routing problem with time windows (VRPTW). For details on the VRPTW, refer to Bräysy and Gendreau (2005a, (2005b) and Kallehauge et al. (2005). In practice, nurses either use their private cars or vehicles provided by the HHC provider. This results in vehicle-related expenses as well as a high demand for parking spots. Furthermore, due to long average service times at clients, vehicles are little utilised throughout the day, especially in urban areas where travel distances between clients are short. Additionally, considerable time is lost looking for parking spots.

Only little work on HHC routing with public transport is found in literature (e.g., Hiermann et al. 2015; Rest and Hirsch 2016). A solution procedure to operate a transport system to deliver and pick up nurses to and from clients and to facilitate the additional option of walking is introduced in Fikar and Hirsch (2015). The problem is modelled as a many-to-many multi-trip dial-a-ride problem (DARP) and results show a high potential to reduce the number of required vehicles. The impact on driving and travel times of nurses when utilising such a trip sharing concept, however, is not investigated. Due to detours, i.e. the transport service has to first pick up or deliver other nurses, and as walking is usually slower than driving, travel times are prone to increase. Additionally, wait times for the services may occur. This shows trade-offs between the cost of increased travel times of nurses and the expense of operating and maintaining a vehicle fleet.

\subsection{Sustainability of HHC transport concepts}

Sustainability is frequently classified in three categories, economic, social and environmental sustainability (UN General Assembly 2005). Services within Europe, which are performed directly at customers' homes or on their premises, for the most part, generate social benefits (Halme et al. 2006). To measure the overall performance, Halme et al. (2006) derive 18 sustainability indicators. Within their category "Care and Supervision", under which HHC is included, social and economic sustainability perform equally well whilst environmental sustainability is lagging behind. 


\subsubsection{Economic factors}

Costs concerning the working time of nurses spent travelling to clients, fixed and variable costs of operating a vehicle fleet and disruption costs in case of delays or failure to reach clients are the main economic factors of HHC transport systems. In particular, driving times are often underestimated by service providers and, therefore, require special consideration (Holm and Angelsen 2014). Furthermore, demand for parking spots results in high opportunity costs for dedicated land, high operation and maintenance costs as well as tax and capital expenses (Shoup 1997), whilst time spent looking for parking spots leads to major increases in nurses' travel times. Concepts based on road transport are further impacted by congestion, which increases not only driving durations, but also distances driven as well as overtime and labour expenses (Figliozzi 2010).

\subsubsection{Social factors}

Concerning mode of transport choices, comfort is often a main argument for driving with separate vehicles (Knuth 2012). Nevertheless, more and more nurses do not have driver permits or are reluctant to drive. This is especially critical to nurses from rural areas who are not familiar with driving in urban settings. Trip sharing enables such nurses to be employed in the HHC industry and reduces driving stress. Additionally, HHC services impact the traffic conditions in local communities. In particular, substantial demand for parking spots occurs due to long vehicle idle times. This either affects HHC providers in case of private parking spots (e.g., to park vehicles over night), or the public, if public parking spots close to clients are occupied by HHC vehicles.

\subsubsection{Environmental factors}

Motorised transport results, among other things, in global, regional and local atmospheric pollution and causes waste, congestion and accidents (Piecyk et al. 2012). In HHC operations, average travel distances between two clients are rather short in both urban and sub-urban settings (Fikar and Hirsch 2015). This results in short vehicle driving durations and high idle times. Consequently, high amounts of start, soak and idle emissions and low average speed occur, which, as shown by Hatzopoulou and Miller (2010), increase air pollution. Beside the distance travelled, fleet composition is an important factor (Lemp and Kockelman 2008). HHC vehicles are either nurses' privately-owned cars or vehicles owned by the service providers. In both cases, these are often inefficient and aged vehicles. The implementation of a transport service can, therefore, incorporate the acquisition of more environmentally friendly vehicles to increase environmental sustainability.

\section{Problem description}

Routing and scheduling of HHC services lead to challenging combinatorial optimisation problems, both for routing with separate cars and with trip and car sharing concepts. In daily $\mathrm{HHC}$ operations, nurses are available to serve client 
requests. Given a complete graph $G=(V, A)$, the vertex set $V=\left\{v_{0}, v_{1}, \ldots, v_{n+1}\right\}$ consists of clients $\left\{v_{1}, \ldots, v_{n}\right\}$ and the depot $\left\{v_{0}, v_{n+1}\right\}$, where all tours of vehicles and nurses start and end. Arcs $(i, j) \in A$ are associated with driving durations $t_{i, j}^{d}$. All clients have to be visited within a time window and, depending on the needs of clients, different nurses can be assigned to each service request. Therefore, nurses have qualification levels and clients have qualification requirements. A nurse is only allowed to serve a client if his/her qualification level is higher or equal to the requirement. Additionally, downgrading of nurses is restricted, i.e. a nurse can only serve a limited number of clients requiring a lower qualification level, to ensure employee satisfaction. Therefore, no downgrading of more than one level is allowed and a maximum of one downgrade per day per nurse is enabled. To perform the service, the nurse is contracted to stay a certain time at the client's home. Over the entire day, nurses' schedules are subject to working as well as break regulations, which include maximum working times and the scheduling of breaks. Depending on the number of client requests and available nurses, not all nurses have to work on a specific day. The objective is to minimise travel times of nurses, which consists of drive and wait times. For a detailed description and a mathematical model of a daily HHC problem, refer to Trautsamwieser et al. (2011). In this setting, each nurse operates a separate vehicles. In contrast, when considering car sharing strategies, the vehicle of a nurse can be used by another nurse if the first nurse is off duty, i.e. before the start and after the end of the first nurse's shift.

To analyse trip sharing concepts and to allow walking, $\operatorname{arcs}(i, j) \in A$ are further associated with walking durations $t_{i, j}^{w}$ and each client in the vertex set $V$ can act both as a pickup and a delivery location. The service provider has vehicles with maximum capacities available to transport nurses between clients. If not utilised, a vehicle returns to the depot and waits for the next service. Detours and maximum wait times of nurses are bounded by maximum durations to ensure nurses' satisfaction. Furthermore, a maximum walking duration between two clients and for an entire walking-route is given. To consider the additional costs of the transport system, working times of drivers spent driving are added to the objective function. For a detailed description of the problem considering trip sharing and the additional option of walking, refer to Fikar and Hirsch (2015).

Figure 1 gives a simplified example of the different concepts ignoring time windows and breaks in order to maintain readability. In the top section of the figure, both nurses use a separate car to visit clients. Nurse 2 visits client 6 with a lower qualification requirement, i.e. the nurse downgrades. Car sharing is implemented in the middle section. After nurse 1 finishes the working day, the same vehicle is used by nurse 2 . Therefore, the start time of nurse 2 is postponed to enable car sharing. Travel times do not increase, however, sharing vehicles is only possible if the shift in start time does not lead to time windows violations. In the bottom section, walking is enabled and nurses share trips at the start and end of the working day. As a consequence, both nurses start and end at the same time. If idle, the transport service returns and waits at the depot. Due to the detours resulting from trip sharing and, as walking is slower than driving, travel times increase, however, instead of two vehicles, only one vehicle is required. 

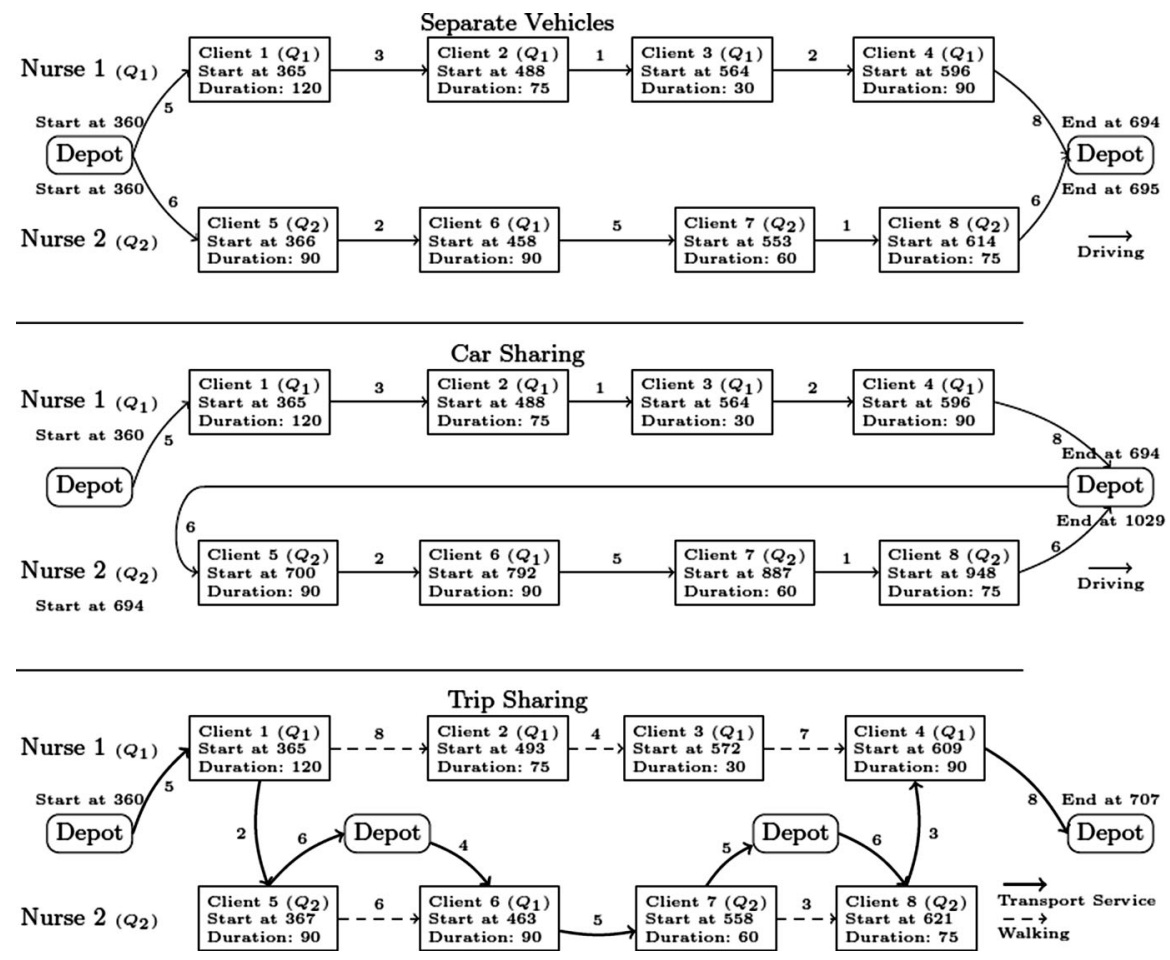

Fig. 1 Nurses' schedules with separate vehicles (top), car sharing (middle) and a transport service (bottom). All time values are in minutes (e.g., $360=6 \mathrm{am}$ ) and two qualification requirements $Q=\{1,2\}$ are given

\section{Methods}

Due to the combinatorial complexity of the underlying optimisation problems, we developed an event-driven biased-randomised heuristic, a post-optimisation procedure and a matheuristic to analyse routing with separate cars, car sharing and trip sharing respectively. These solution procedures generate nurses' schedules and vehicle routes, which are evaluated to perform the comparison. The following subsections briefly review these solution procedures.

\subsection{Routing with separate cars}

Expanding on the ideas presented in Fikar et al. (2016), an event-driven randomised heuristic to derive nurses' schedules and vehicle routes for concepts where each nurse uses a separate vehicle was developed. Therefore, a wide range of promising solutions is generated within a short time-frame. To speed up computation, this is done in parallel on multiple threads of the operating system. Nurses are assigned randomly to certain clients to start their working days. Whenever a nurse requires a new routing or scheduling decision, an event is called to decide which client a nurse is scheduled to serve next. 
Therefore, marginal costs, which consider the distance of the nurse to the client as well as the distance of the closest other nurse to the same client, are considered. Then, all potential clients are added to a list and sorted by the respective value. Biased-randomisation using a geometric distribution (Juan et al. 2013) selects one client, whereas less costly clients are given a higher probability. Therefore, the parameter $\beta$ in the range of $0-1$ indicates the bias of the selection, whereas a low $\beta$ value indicates higher randomness in the selection. This enables a high number of different solutions within multiple runs. For our test runs, $\beta$ is uniformly randomly selected for each construction between 0.5 and 1. Feasibility checks concerning time windows, qualification requirements as well as break times and working time regulations are performed constantly during the runs. In case of a violation, the current solution is neglected and the algorithm continues with the construction of a new solution. Feasible solutions are further improved with a local search and a memory function. Therefore, the former tests solutions for improvement by relocating a job, whilst the latter saves complete routes and exchanges these in a single solution if a better route with the same stops was previously found. The procedure, summarised in Algorithm 1, stops after 1 min of run time. It was benchmarked with the mixed-integer problem formulation provided in Trautsamwieser et al. (2011) and the solver software FICO Xpress 7.8. Therefore, test instances with 20 clients and four nurses were generated. The procedure reaches the optimum on 19 of the 20 problem instances, while the solution is 1 min worse on the remaining instance, resulting in an average gap of $0.01 \%$.

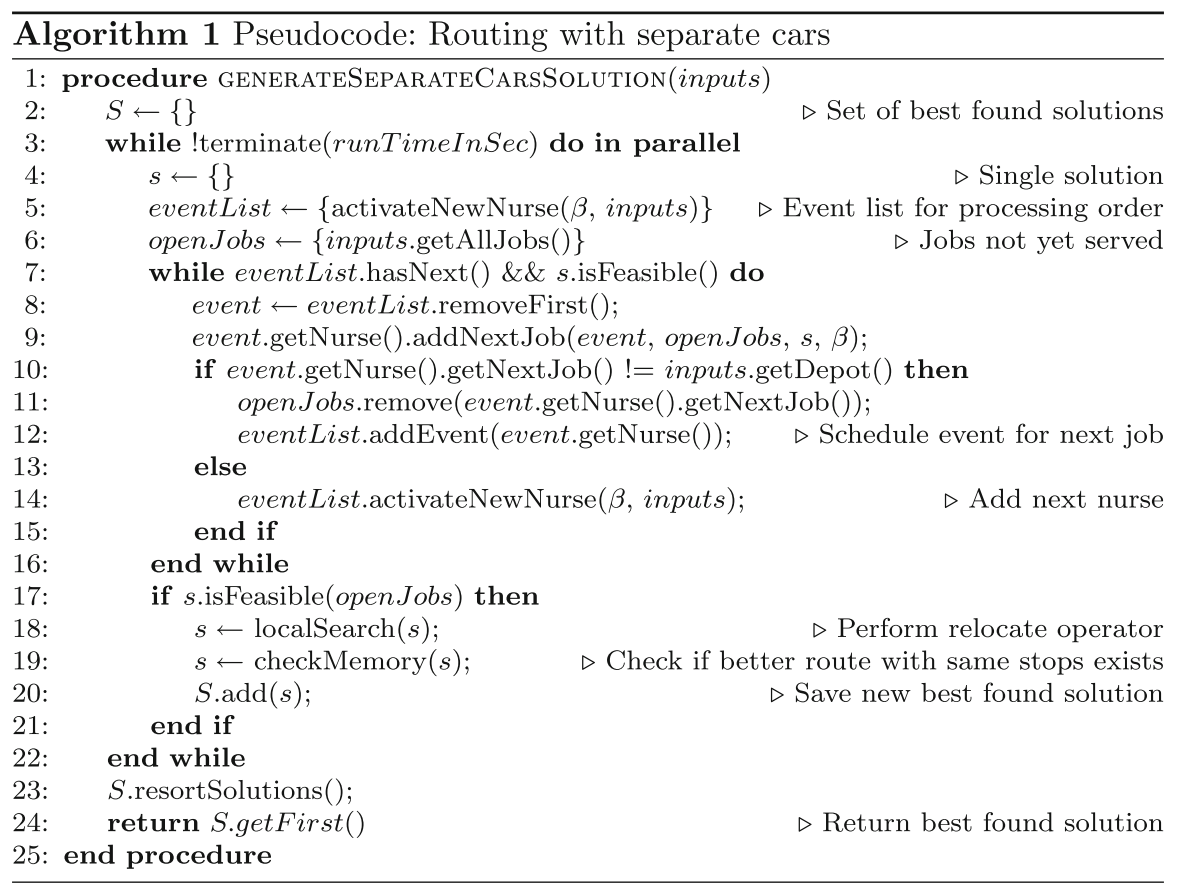

\subsection{Implementing a car sharing system}


Based on the generated solutions for routing with separate cars, implementation of a car sharing concept enables HHC planers to reduce the number of required vehicles. Therefore, a post-optimisation heuristic was developed, which tests if nurses can share vehicles throughout the day based on the solution generated by Algorithm 1 . To increase flexibility, start times of nurses are altered based on the forward slack time (Savelsbergh 1992), which indicates how much the start time can be delayed without violating time windows. As a result, nurses who can share a vehicle are identified by the solution procedure, e.g., a nurse performing a morning shift shares a vehicle with a nurse working in the afternoon. The procedure starts with sorting all nurses based on the end time of the nurse's shift. Starting with the first nurse on the list, all nurses are tested to determine whether car sharing is feasible. In some cases, this requires shifting start times, which is enabled by the calculated forward slack to a maximum value without increasing wait times. This procedure is repeated until no more vehicles can be shared. The complete procedure is explained in Algorithm 2.

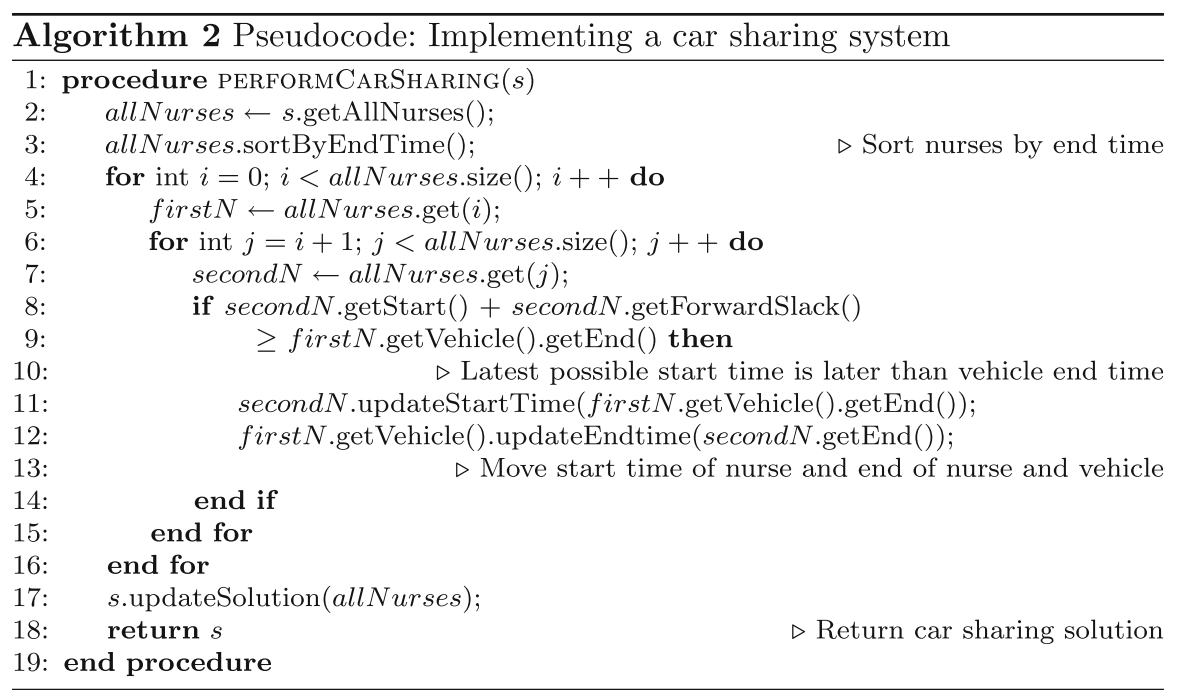

\subsection{Routing trip sharing transport systems}

To route and schedule trip sharing concepts facilitating walking, the algorithm has to decide when a nurse walks and when the transport service is utilised. Furthermore, it has to guarantee synchronisation of nurses and vehicles. For instance, a nurse who starts a service at $1 \mathrm{pm}$, which requires $1 \mathrm{~h}$ for completion, has to be picked up starting from $2 \mathrm{pm}$. Each minute after $2 \mathrm{pm}$ results in waiting for the nurse, which leads to additional costs. For trip sharing, three different metaand matheuristic solution procedures were developed in previous work (Fikar and Hirsch 2015; Fikar et al. 2016). The matheuristic TS-SPBS (Fikar and Hirsch 2015) 
was selected for this work as it performs best in respect to solution quality. We extended this solution procedure, summarized in Algorithm 3, to consider different objective functions.

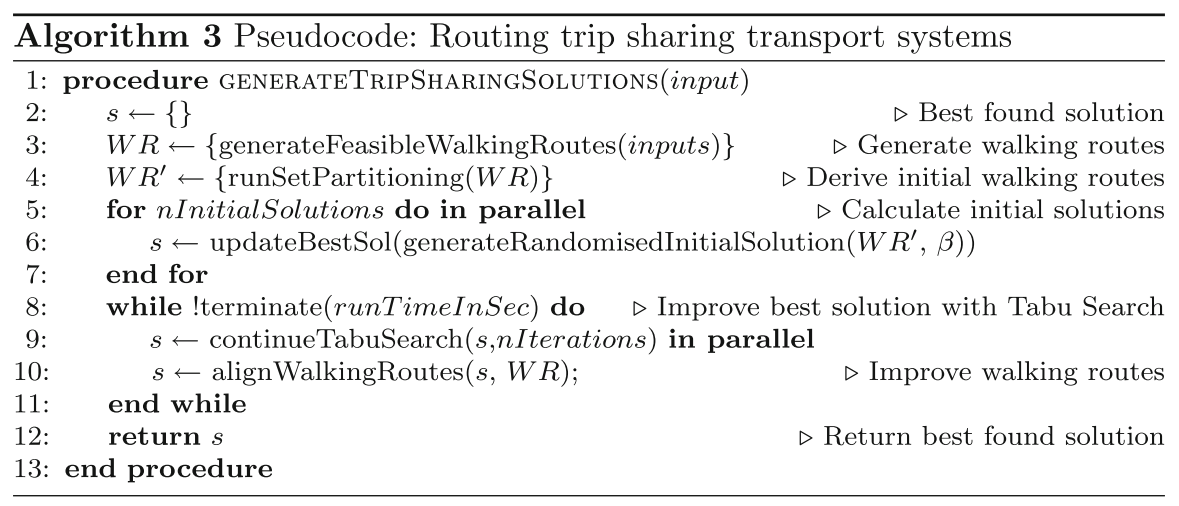

Walking-routes are created by a set partitioning procedure and, based on this set of walking-routes, multiple initial solutions are constructed. The best result is then further improved by a Tabu Search metaheuristic. Wait times are minimised by running a linear programme to optimise start times of vehicles. Furthermore, at certain points during the run, walking-routes are modified to align walking and vehicle routes. Therefore, a change in the sequence of clients within a walking-route is tested as well as splitting and merging routes. Due to the time-consuming exact components, the algorithm runs for $1 \mathrm{~h}$ for each instance. Parameters are set in accordance to the test settings in Fikar and Hirsch (2015) and the best results of ten test runs are reported.

\subsection{Experimental design}

For computational experiments, we use test instances provided in Fikar and Hirsch (2015). The 100-client sized instances are presented in this paper whilst additional material covering the 75 and 125-sized instances is available online at http://www. wiso.boku.ac.at/en/production-and-logistics/research/instances/. These instances are derived from real-world geographic locations of clients and consider road networks of the respective areas. A walking speed of $3.6 \mathrm{~km} / \mathrm{h}$ is assumed. Five instances are located in an urban area and five in a sub-urban area, indicated in the instance names with "U" and "S" respectively. The geographic position of clients is changed for each instance. Parking delays are included by increasing the driving durations for all trips to client locations by $p$ when using separate cars. Nurses (service requests) can have three different qualification levels (requirements). In the first qualification level, which represents the lowest level, 16 nurses are available, 10 in the second and 6 in the third. Within a day, each nurse is allowed to serve a maximum of one client one level below the nurse's qualification level. Maximum working time per day is $10 \mathrm{~h}$. If a nurse works longer than $6 \mathrm{~h}$, a break of $30 \mathrm{~min}$ has to be scheduled. 
This break is not counted in the working time and has to be scheduled so that the nurse does not work longer than $6 \mathrm{~h}$ consecutively. To analyse the trip sharing concept, two vehicles with a capacity of six including the driver are available. Maximum walking distances are constrained by $10 \mathrm{~min}$ between two clients and by 20 min between two trips on the transport service. Wait times between delivery and pickup as well as additional ride times per trip due to detours are limited to $15 \mathrm{~min}$. For each area, the same five settings of time windows, qualification levels and service durations are applied. These originated from statistical distributions of realworld operations and are summarised in Table 1.

To further analyse the impact of the geographic distribution of clients, we adopt the Solomon instances of group one with 100 clients (Solomon 1987). These focus on short scheduling horizons as commonly present in daily HHC operations and were acquired from http://w.cba.neu.edu/ msolomon/problems.htm. Three categories of client distributions are represented. Clusters of clients are present in "C"instances, a setting often found in commuter towns outside of a city as well as in rural areas. In instances indicated by " $R$ ", clients are distributed randomly over the test region, similar to urban city centres. Instances "RC" include a combination of randomly distributed clients and clusters as often seen in suburbs. Figure 2 plots each type of instances. To adjust the instances to HHC operations, we only take the client locations from the Solomon instances. As coordinates are given, we further take the Euclidean distances as walking duration and divide them by a factor of 10 to calculate driving durations. All durations were rounded up to the next integer value. As the different Solomon instances for each category do not differ in coordinates, we only calculate one geographic distribution per category. For each geographic distribution, two different test settings are investigated. First, the five different time window, service duration and qualification requirement settings from Table 1 are utilised. These instances are denoted with R-HHC, C-HHC and RCHHC. Second, to indicate the impact of time windows, qualification requirements and service durations, we further perform the test runs considering only one qualification level, no time windows and a fixed service duration of $1 \mathrm{~h}$. These instances are denoted as R'-HHC, C'-HHC and RC'-HHC.

To investigate the impact of different objectives of decision makers and resulting trade-offs, we further run the $\mathrm{U}$ and $\mathrm{S}$ instances with different objective functions. These either only reduce the drive time (1) or the travel times of nurses (2), which consist of durations walked, waited and spent on board a vehicle, of a solution $S$ and are compared to optimising the sum of drive and travel times (3) as considered in the other experiments.

$$
\begin{gathered}
\text { minimise } f(S)=S_{\text {Drive }} \\
\text { minimise } f(S)=S_{\text {Walk }}+S_{\text {Wait }}+S_{\text {Onboard }} \\
\text { minimise } f(S)=S_{\text {Drive }}+S_{\text {Walk }}+S_{\text {Wait }}+S_{\text {Onboard }}
\end{gathered}
$$

Additionally, we investigate a fourth option (4) by adding a weight $\alpha \in[0,1]$ to the objective function. This allows the decision maker to either put a weight on drive 


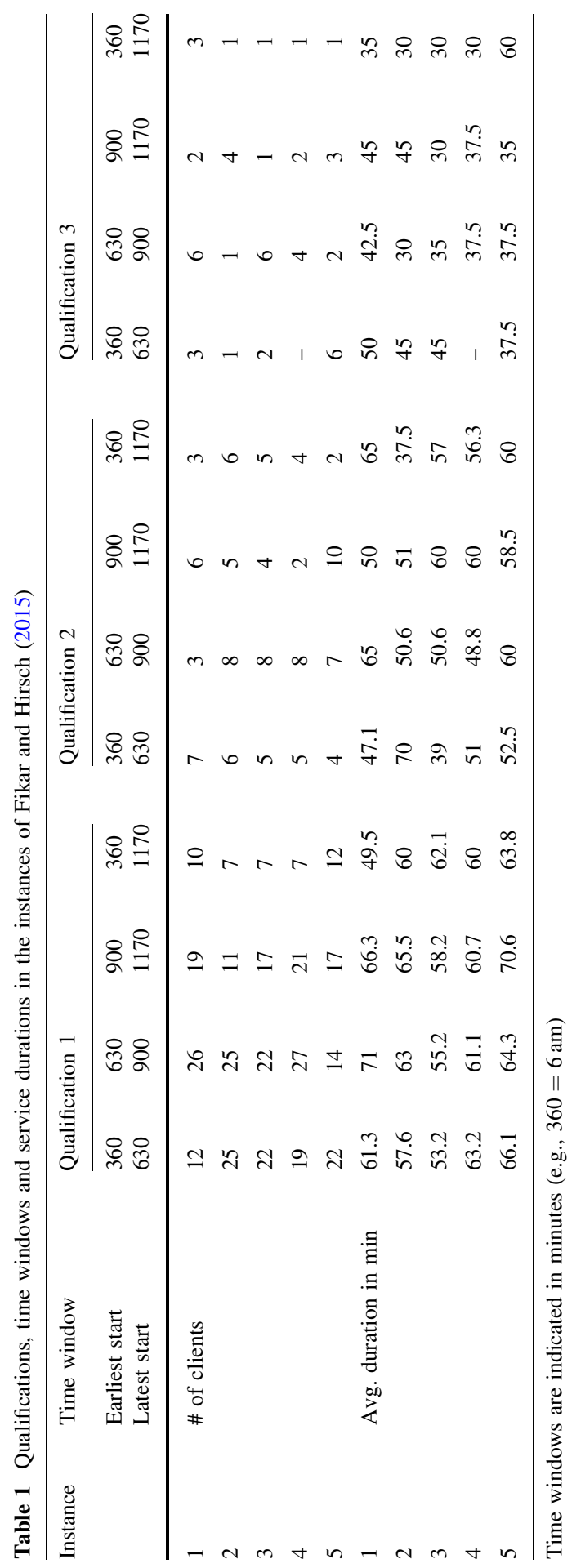




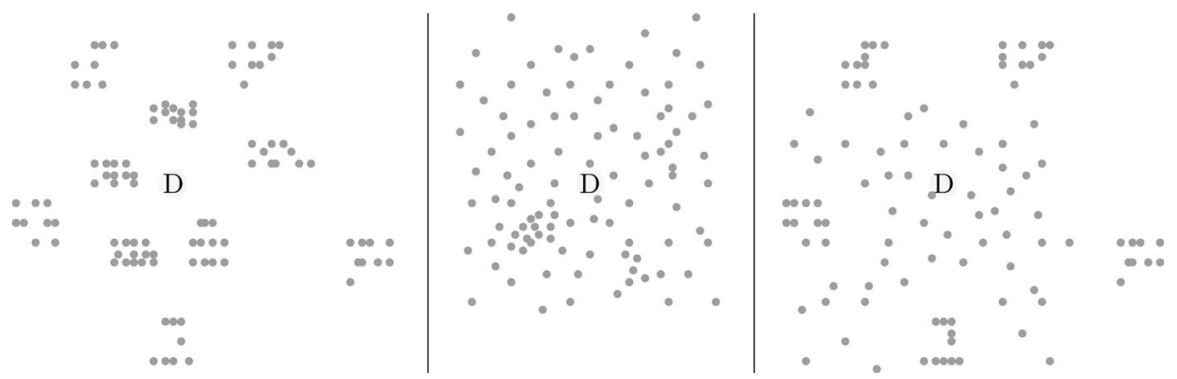

Fig. 2 Geographic distribution of clients. Plots of each type, C (left), R (middle) and RC (right). "D" indicates the depot, dots the locations of clients

times, which are multiplied by $\alpha$, or on nurses' travel times, which are evaluated with $1-\alpha$.

$$
\text { minimise } f(S)=\alpha S_{\text {Drive }}+(1-\alpha)\left(S_{\text {Walk }}+S_{\text {Wait }}+S_{\text {Onboard }}\right)
$$

The experiments give implications on the impact of the studied factors, however, the following research limitations and assumptions have to be considered. This work is based on organisational frameworks and national regulations in Austria. The comparison is based on a static problem setting as well as matheuristic and metaheuristic solution procedures. Therefore, optimal solutions may deviate, however, general implications of this work are expected to remain valid based on benchmarks as well as various test scenarios and settings. Additionally, the impact of uncertainties and stochasticies is little studied in literature for routing with separate vehicles and no work on stochastic routing and scheduling with trip sharing concepts exists. Nevertheless, relevant stochastic factors, which may be independent or interdependent and potentially impact the performance include stochastic travel times, service delays and varying driver behaviours.

\section{Results and discussion}

In this section, computational results are first compared to classical concepts where each nurse operates a separate vehicle. In the next step, prerequisites for a successful implementation of these concepts are analysed by investigating different geographic distributions of customers. Lastly, the importance of the main objective of the decision maker is analysed. All test runs were gathered on an Intel Core i7-4930K, 64GB RAM, with Windows 7 as operating system and 6 threads operating in parallel.

\subsection{Impact of trip sharing}

Table 2 compares the trip sharing concept, denoted in the following part as TripSharing, with operations where each nurse uses a separate vehicle, denoted as allCars. CarSharing states the setting where multiple nurses in allCars share a 
Table 2 Comparison of TripSharing, allCars and CarSharing on urban and sub-urban real-world based instances

\begin{tabular}{|c|c|c|c|c|c|c|}
\hline & \multicolumn{3}{|c|}{ Travel time nurses } & \multirow{2}{*}{$\begin{array}{l}\text { Drivers } \\
\text { TripSharing }\end{array}$} & \multicolumn{2}{|c|}{$\#$ of vehicles $(+/-)$} \\
\hline & allCars & TripSharing & $+/-(\%)$ & & CarSharing (\%) & TripSharing (\%) \\
\hline U1-N100 & 217 & 401 & 84.8 & 301 & -31.3 & -87.5 \\
\hline U2-N100 & 212 & 404 & 90.6 & 303 & -25.0 & -87.5 \\
\hline U3-N100 & 199 & 387 & 94.5 & 313 & -23.1 & -84.6 \\
\hline U4-N100 & 209 & 382 & 82.8 & 308 & -20.0 & -86.7 \\
\hline U5-N100 & 222 & 401 & 80.6 & 335 & -35.3 & -88.2 \\
\hline S1-N100 & 297 & 477 & 60.6 & 550 & -33.3 & -88.9 \\
\hline S2-N100 & 282 & 484 & 71.6 & 514 & -33.3 & -88.9 \\
\hline S3-N100 & 271 & 452 & 66.8 & 587 & -26.7 & -86.7 \\
\hline S4-N100 & 279 & 483 & 73.1 & 500 & -31.6 & -89.5 \\
\hline S5-N100 & 307 & 462 & 50.5 & 634 & -42.1 & -89.5 \\
\hline
\end{tabular}

vehicle. Concerning travel time, CarSharing equals allCars. Travel time considers time spent by nurses on board a vehicle, walked or waited.

The results show that TripSharing leads to a drastic increase in travel times of up to $95 \%$ in urban and $73 \%$ in sub-urban areas. This is due to detours resulting from nurses being delivered and picked up, as wait times for the transport service occur and walking is slower than driving (exceptions occur for one-way streets and designated pedestrian areas). Furthermore, such an increase in travel and wait times may require providers to employ more nurses in TripSharing. Nevertheless, the results show that TripSharing leads to a major decrease of about $88 \%$ in the number of required vehicles. Additionally, longer service times at clients' homes lead to lower vehicle utilisation in allCars as less distance is travelled on a working day. As a consequence, the potential reduction in vehicles of TripSharing is higher in such situations. Concerning the number of required vehicles for TripSharing, the number of clients to serve, average distances between homes and the amount of potential walking is of importance. HHC providers can significantly reduce expenses for leasing, vehicle-related taxes, insurances, maintenance and parking if the vehicles are operated by the provider. Furthermore, in such a setting, HHC providers can alternatively facilitate CarSharing, allowing one to reduce the number of required vehicles between 20 and $42 \%$ without increasing travel times. Long-term savings of TripSharing have to be weighted by the decision maker with the daily increases in variable costs for additional time spent to travel to clients, the impact on fuel consumptions and the costs of hiring drivers for the transport services. If nurses are using their private cars, mileage reimbursements are avoided, which again has to be compared against the additional costs of TripSharing.

One benefit of implementing TripSharing is that is does not require parking spots at client locations whilst the service is performed. Therefore, substantial time to look for a parking spot, to park and to walk from the potentially distant parking spot to the client can be saved. Planning algorithms often do not consider this 
complication of real-world operations. Table 3 shows the impact of parking delays on the travel time of nurses. In urban areas, TripSharing leads to a reduction in the total duration driven if a parking delay of only $1 \mathrm{~min}$ is considered, however, travel times of nurses are still increased. Travel times of nurses in both urban and suburban instances are reduced starting with a parking delay of $2 \mathrm{~min}$, whilst driving durations in sub-urban instances are still increased. With a parking delay of $3 \mathrm{~min}$, TripSharing outperforms allCars in eight out of ten test settings considering both travel times and driving durations. This shows the potential of trip sharing in settings where parking issues are present.

\subsection{Geographic distribution of clients}

Understanding how TripSharing performs in different geographic distributions of clients enables providers to find areas for implementation and indicates strengths and weaknesses of the compared concepts. The average percentages of the objective value, which are driven, walked, spent by nurses on board a vehicle and waited are given in Table 4 for the different geographic distributions. Walking is especially beneficial in settings where clients are clustered. Having multiple clients close to each other allows the nurse to walk to the subsequent clients without requiring the transport service. This further helps other nurses, as the transport service is less utilised, resulting in lower wait times and fewer detours.

Table 5 compares TripSharing and CarSharing with allCars.Long delays occur if nurses are required to travel between clusters. In randomly distributed areas, nurses can walk in case the transport service is not available, however, walking is costly as average distances are long. The combination of clusters and randomly distributed clients leads to the best results for TripSharing concerning nurses' travel times as disadvantages of both are mitigated. Nurses can efficiently walk within clusters, however, if a nurse completed all assignments within a cluster and requesting a vehicle is costly, the nurse usually still has potential clients within walking distance. Concerning CarSharing, over all geographic distributions, the number of required vehicles can be substantially reduced without increasing travel times, however, not to the same extent as TripSharing. Additionally, Table 6 presents the results if no time windows, only one qualification level and a fixed service duration are assumed. The experiments show that time windows, qualification levels and different service durations have a substantial impact on the performance of TripSharing as they limit potential walking options and further complicate synchronisation of nurses and vehicles for pickups. More flexibility in the timing decision allows for better sharing of trips. This results in lower driving durations and more efficient routes. Nevertheless, allCars also profits from this flexibility, increasing the gap between the two concepts.

\subsection{Objective of the decision maker}

Due to detours, TripSharing may lead to an increase in the total distance driven. Nevertheless, if trips are shared well, driving distances may even decrease as certain paths are shared by multiple nurses. Therefore, the decision maker has to specify an 
Table 3 Impact of time spent for parking $(p)$ on allCars compared to TripSharing

\begin{tabular}{|c|c|c|c|c|c|c|}
\hline & \multicolumn{2}{|l|}{$p=1$} & \multicolumn{2}{|l|}{$p=2$} & \multicolumn{2}{|l|}{$p=3$} \\
\hline & $\begin{array}{l}+/- \\
\text { driven }(\%)\end{array}$ & $\begin{array}{l}\text { Travel time } \\
\text { nurses }(\%)\end{array}$ & $+/-$ driven $(\%)$ & $\begin{array}{l}\text { Travel time } \\
\text { nurses }(\%)\end{array}$ & $+/-$ driven $(\%)$ & $\begin{array}{l}\text { Travel time } \\
\text { nurses }(\%)\end{array}$ \\
\hline U1-N100 & 5.3 & -20.9 & 39.2 & 4.5 & 72.4 & 29.4 \\
\hline U2-N100 & 2.3 & -23.3 & 36.3 & 2.2 & 70.0 & 27.5 \\
\hline U3-N100 & -3.8 & -22.2 & 29.1 & 4.4 & 61.0 & 30.2 \\
\hline U4-N100 & 0.6 & -18.8 & 33.4 & 7.6 & 65.9 & 33.8 \\
\hline U5-N100 & -5.1 & -20.7 & 24.5 & 4.0 & 54.9 & 29.4 \\
\hline S1-N100 & -28.4 & -17.4 & -9.3 & 4.6 & 7.5 & 23.9 \\
\hline S2-N100 & -26.5 & -21.9 & -7.0 & -1.2 & 13.4 & 20.5 \\
\hline S3-N100 & -35.4 & -16.2 & -18.6 & 5.8 & -1.4 & 28.1 \\
\hline S4-N100 & -25.0 & -22.4 & -4.4 & -1.0 & 15.8 & 19.9 \\
\hline S5-N100 & -35.6 & -11.7 & -19.1 & 11.0 & -3.2 & 32.9 \\
\hline
\end{tabular}

Table 4 Average percentage of the TripSharing objective value spent for driving, walking, on board a vehicle and waiting

\begin{tabular}{lllll}
\hline & Driver & \multicolumn{2}{l}{ Nurse } & \\
\cline { 3 - 5 } & \% Driven & $\%$ Walked & \% On board & \% Waited \\
\hline C-HHC & 44.8 & 27.9 & 26.9 & 0.5 \\
R-HHC & 55.7 & 17.8 & 25.4 & 1.1 \\
RC-HHC & 54.0 & 17.8 & 27.0 & 1.2 \\
\hline
\end{tabular}

objective to deal with this trade-off. Whilst reducing drive times of vehicles results in environmentally sustainable solutions, decreasing the travel time of nurses, depending on the cost structure of the HHC provider, potentially results in more financially sustainable solutions. Tables 7 and 8 show the impact of both only reducing drive times and only reducing travel times of nurses compared to optimising the sum of drive and travel times as described in the previous subsections.

The results indicate that substantial reductions in the driving distances of nearly $50 \%$ are achievable by TripSharing, however, it highly impacts nurses' travel times. If the main focus is to reduce travel times, driving duration nearly doubles. Figure 3 plots these different objectives by increasing the weight $\alpha$ in increments of 0.1 . If $\alpha$ is 0 , drive times of the vehicles are completely ignored, if 1 , travel times of nurses are not considered. The results indicate that the sum of driving durations and travel times of nurses shows a convex behaviour. Slightly deviating from the minimum only leads to minor changes in the sum of durations. This allows the decision maker to substitute between reducing the duration driven and the time travelled by nurses. Additionally, the results indicate that these small changes mostly affect the duration walked or driven. This allows direct substitution between these two modes of transport and the resulting costs for either drivers or nurses, 
Table 5 Comparison of TripSharing, allCars and CarSharing in different geographic distributions of clients

\begin{tabular}{lllllll}
\hline & \multicolumn{2}{l}{ Travel time nurses } & & Drivers & \multicolumn{2}{l}{ \# of vehicles $(+/-)$} \\
\cline { 2 - 3 } & allCars & TripSharing & $+/-(\%)$ & TripSharing & CarSharing $(\%)$ & TripSharing $(\%)$ \\
\hline C1-HHC & 253 & 422 & 66.8 & 369 & -23.5 & -88.2 \\
C2-HHC & 223 & 408 & 83.0 & 325 & -44.4 & -88.9 \\
C3-HHC & 241 & 414 & 71.8 & 323 & -35.3 & -88.2 \\
C4-HHC & 244 & 410 & 68.0 & 329 & -25.0 & -87.5 \\
C5-HHC & 244 & 463 & 89.8 & 371 & -35.3 & -88.2 \\
R1-HHC & 220 & 390 & 77.3 & 461 & -35.3 & -88.2 \\
R2-HHC & 218 & 377 & 72.9 & 477 & -37.5 & -87.5 \\
R3-HHC & 210 & 382 & 81.9 & 479 & -37.5 & -87.5 \\
R4-HHC & 213 & 371 & 74.2 & 485 & -36.8 & -89.5 \\
R5-HHC & 229 & 379 & 65.5 & 487 & -45.0 & -90.0 \\
RC1-HHC & 267 & 448 & 67.8 & 468 & -40.0 & -90.0 \\
RC2-HHC & 266 & 391 & 47.0 & 512 & -33.3 & -88.9 \\
RC3-HHC & 254 & 412 & 62.2 & 508 & -20.0 & -86.7 \\
RC4-HHC & 254 & 440 & 73.2 & 453 & -25.0 & -87.5 \\
RC5-HHC & 270 & 422 & 56.3 & 536 & -33.3 & -88.9 \\
\hline
\end{tabular}

whilst the duration spent on board is more stable. Nevertheless, if reducing the duration driven is of high focus, travel times start to dramatically increase as major wait times occur. In sub-urban areas, substitution between different modes is more difficult to achieve due to longer distances between clients, leading to flatter functions.

\section{Conclusions}

This work compared a trip sharing concept facilitating walking to current practices and analysed the concept's performance in different geographic client distributions and under different objectives of decision makers. Furthermore, a car sharing

Table 6 Comparison of TripSharing with allCars ignoring time windows, qualifications and with a fixed service duration

\begin{tabular}{|c|c|c|c|c|c|c|}
\hline & \multicolumn{3}{|c|}{ Travel time nurses } & \multirow{2}{*}{$\begin{array}{l}\text { Driver } \\
\text { TripSharing }\end{array}$} & \multicolumn{2}{|c|}{$\#$ of Vehicles $(+/-)$} \\
\hline & allCars & TripSharing & $+/-(\%)$ & & CarSharing (\%) & TripSharing (\%) \\
\hline $\mathrm{C}^{\prime}-\mathrm{HHC}$ & 163 & 343 & 110.4 & 169 & 0.0 & -83.3 \\
\hline $\mathrm{R}^{\prime}-\mathrm{HHC}$ & 157 & 328 & 108.9 & 368 & 0.0 & -83.3 \\
\hline RC'-HHC & 179 & 392 & 119.0 & 298 & 0.0 & -83.3 \\
\hline
\end{tabular}


strategy was considered. Therefore, solution procedures for the different concepts were developed or extended. According to the computational experiments, trip sharing in HHC services performs best in areas where long service times and challenges to find parking spots occur, leading to a substantial reduction in the number of required vehicles. Furthermore, areas where clients are both geographically clustered and randomly distributed show greater potential for the implementation of such concepts according to our evaluation. Nevertheless, additional drivers have to be compensated and travel durations are prone to increase compared to the usage of separate vehicles due to detours, potentially increasing the number of nurses required on a given day of operation. In contrast, car sharing enables HHC providers to reduce the number of required vehicles without an increase in travel times, however, not to the extent of trip sharing. Furthermore, it does not require the acquisition of dedicated professional drivers and transport vehicles, making this option especially interesting for smaller HHC providers who are unable to implement trip sharing strategies. Even though this analysis focuses on HHC services, results can be transferred to different home service industries where similar challenges and long service times occur.

Additionally, implementing trip sharing concepts potentially improves employee satisfaction by lowering driving stress and can lead to environmentally friendlier operations if driving durations are reduced and walking is facilitated. Nevertheless, trade-offs between the costs of operating a large fleet of individually operated vehicles and the additional costs of operating a trip sharing transport system need to be considered closely. Additionally, daily operations are potentially prone to disruptions and delays, which may have a major impact on the benefits of trip and car sharing. Therefore, decision makers have to carefully analyse their services and operational area as well as resulting risks and impacts on patient and staff satisfaction before introducing such concepts.

Although we have extensively investigated trip and car sharing using both realworld based and theoretic data, future work and field tests are required to

Table 7 Comparison of minimising drive durations $(\alpha=1)$ and optimising both travel times of nurses and driver $(\alpha=0.5)$

\begin{tabular}{lllll}
\hline & $+/-$ driver $(\%)$ & $+/-$ walk $(\%)$ & $\begin{array}{l}+/- \text { Nurse on } \\
\text { board/waiting }(\%)\end{array}$ & $\begin{array}{l}\text { Total travel } \\
\text { time nurses }(\%)\end{array}$ \\
\hline U1-N100 & -44.9 & 81.5 & 175.9 & 131.4 \\
U2-N100 & -45.9 & 60.8 & 219.8 & 136.2 \\
U3-N100 & -49.8 & 80.8 & 206.4 & 133.7 \\
U4-N100 & -45.8 & 90.8 & 200.0 & 132.4 \\
U5-N100 & -46.9 & 75.4 & 228.4 & 149.4 \\
S1-N100 & -36.0 & 50.0 & 262.7 & 234.9 \\
S2-N100 & -37.4 & 72.8 & 214.9 & 220.2 \\
S3-N100 & -37.0 & 66.0 & 315.1 & 272.8 \\
S4-N100 & -32.6 & 41.3 & 275.9 & 235.6 \\
S5-N100 & -37.7 & 105.7 & 256.5 & 270.8 \\
\hline
\end{tabular}


Table 8 Comparison of minimising nurse travel times $(\alpha=0)$ and optimising both travel times of nurses and driver $(\alpha=0.5)$

\begin{tabular}{lllcl}
\hline & $+/-$ driver $(\%)$ & $+/-$ walk $(\%)$ & $\begin{array}{c}+/- \text { Nurse on } \\
\text { board/waiting }(\%)\end{array}$ & $\begin{array}{l}\text { Total travel } \\
\text { time nurses }(\%)\end{array}$ \\
\hline U1-N100 & 164.8 & -90.0 & 7.1 & -38.7 \\
U2-N100 & 154.1 & -87.6 & 23.0 & -35.9 \\
U3-N100 & 120.1 & -83.3 & 8.0 & -40.9 \\
U4-N100 & 132.5 & -86.2 & 19.5 & -37.9 \\
U5-N100 & 133.1 & -89.9 & 17.5 & -37.9 \\
S1-N100 & 80.2 & -86.8 & 3.5 & -18.0 \\
S2-N100 & 91.3 & -89.4 & -1.0 & -17.7 \\
S3-N100 & 66.6 & -87.6 & 4.4 & -17.5 \\
S4-N100 & 97.6 & -92.0 & 12.1 & -17.2 \\
S5-N100 & 69.2 & -87.8 & -4.8 & -12.2 \\
\hline
\end{tabular}
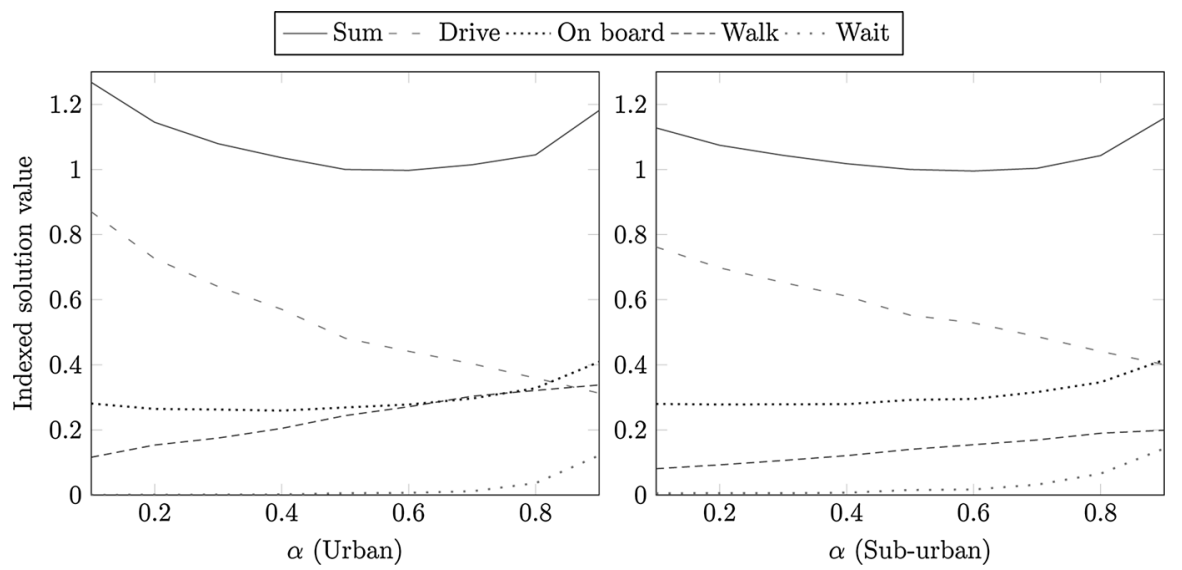

Fig. 3 Trade-offs between driving and nurses' travel times. Average results with $\alpha=\{0.1,0.2, \ldots, 0.9\}$ for U1-N100 to U5-N100 (left) and S1-N100 to S5-N100 (right). All values are indexed to the sum of durations with $\alpha=0.5$

comprehensively analyse the impact on real-world implementations. Most work on HHC routing and scheduling ignores stochasticities and uncertainties. Potential challenges are, e.g., cancellations, additional client requests or disruptions of daily schedules due to delays in travel or service times. This offers a wide range of research questions, which need to be explored to better understand potential disruption risks. Additionally, extending our work by performing cost-utility analyses or facilitating sustainability indicators is of high interest for HHC services as well as other related industries. Furthermore, the impact of trip and car sharing on patient and nurse satisfaction has to be closely analysed. Studies surveying both groups would contribute to the acceptance of future implementations in HHC operations. 
Acknowledgments Open access funding provided by University of Natural Resources and Life Sciences Vienna (BOKU). We thank the Austrian Red Cross, especially Monika Wild, Johanna Reinisch, Reinhard Schmidt and Harald Pfertner, for providing us with data and feedback.

Open Access This article is distributed under the terms of the Creative Commons Attribution 4.0 International License (http://creativecommons.org/licenses/by/4.0/), which permits unrestricted use, distribution, and reproduction in any medium, provided you give appropriate credit to the original author(s) and the source, provide a link to the Creative Commons license, and indicate if changes were made.

\section{References}

ADB (2010) Sustainable transport initiative operational plan. Asian Development Bank, Manila, Philippines

Begur SV, Miller DM, Weaver JR (1997) An integrated spatial DSS for scheduling and routing homehealth-care nurses. Interfaces 27(4):35-48. doi:10.1287/inte.27.4.35

Bräysy O, Gendreau M (2005a) Vehicle routing problem with time windows, part I: route construction and local search algorithms. Transp Sci 39(1):104-118. doi:10.1287/trsc.1030.0056

Bräysy O, Gendreau M (2005b) Vehicle routing problem with time windows, part II: metaheuristics. Transp Sci 39(1):119-139. doi:10.1287/trsc.1030.0057

Cappanera P, Scutellá MG (2015) Joint assignment, scheduling, and routing models to home care optimization: a pattern-based approach. Transp Sci. doi:10.1287/trsc.2014.0548

Chahed S, Marcon E, Sahin E, Feillet D, Dallery Y (2009) Exploring new operational research opportunities within the home care context: the chemotherapy at home. Health Care Manag Sci 12(2):179-191. doi:10.1007/s10729-009-9099-6

Dorin L, Turner S, Beckmann L, Große Schlarmann J, Faatz A, Metzing S, Buscher A (2014) Which need characteristics influence healthcare service utilization in home care arrangements in Germany? BMC Health Serv Res 14(1):233. doi:10.1186/1472-6963-14-233

EC (2011) White paper-roadmap to a single European transport area-towards a competitive and resource efficient transport system. European Commission, 144 final, Brussels, Belgium

Ferguson E (1997) The rise and fall of the American carpool: 1970-1990. Transportation 24(4):349-376. doi:10.1023/A:1004928012320

Figliozzi MA (2010) The impacts of congestion on commercial vehicle tour characteristics and costs. Transp Res E Log Transp Rev 46(4):496-506. doi:10.1016/j.tre.2009.04.005

Fikar C, Hirsch P (2015) A matheuristic for routing real-world home service transport systems facilitating walking. J Clean Prod 105:300-310

Fikar C, Juan A, Martinez E, Hirsch P (2016) A discrete-event driven metaheuristic for dynamic home service routing with synchronised trip sharing. Eur J Ind Eng 10:3. doi:10.1504/EJIE.2016.076382

Furuhata M, Dessouky M, Ordóñez F, Brunet ME, Wang X, Koenig S (2013) Ridesharing: the state-ofthe-art and future directions. Transp Res B Methodol 57:28-46. doi:10.1016/j.trb.2013.08.012

Halme M, Anttonen M, Hrauda G, Kortman J (2006) Sustainability evaluation of European household services. J Clean Prod 14(17):1529-1540. doi:10.1016/j.jclepro.2006.01.021

Hatzopoulou M, Miller E (2010) Linking an activity-based travel demand model with traffic emission and dispersion models: transport's contribution to air pollution in Toronto. Transp Res D Trans Environ 15(6):315-325. doi:10.1016/j.trd.2010.03.007

Hiermann G, Prandtstetter M, Rendl A, Puchinger J, Raidl G (2015) Metaheuristics for solving a multimodal home-healthcare scheduling problem. Cent Eur J Oper Res 23(1):89-113. doi:10.1007/ s10100-013-0305-8

Holm S, Angelsen R (2014) A descriptive retrospective study of time consumption in home care services: how do employees use their working time? BMC Health Serv Res 14(1):439. doi:10.1186/14726963-14-439

Juan A, Faulin J, Ferrer A, Lourenço H, Barrios B (2013) MIRHA: multi-start biased randomization of heuristics with adaptive local search for solving non-smooth routing problems. Top 21(1):109-132. doi:10.1007/s11750-011-0245-1 
Kallehauge B, Larsen J, Madsen O (2005) Vehicle routing problem with time windows. In: Desaulniers G, Desrosiers J, Solomon M (eds) Column generation. Springer, Berlin, pp 67-98. doi:10.1007/0387-25486-2_3

Knuth KR (2012) Factors influencing behavioural change towards eco-friendly multimodal mobility. EU Project USEmobility, Deliverable D3.6

Lemp JD, Kockelman KM (2008) Quantifying the external costs of vehicle use: evidence from America's top-selling light-duty models. Transp Res D Transp Environ 13(8):491-504. doi:10.1016/j.trd.2008. 09.005

Matta A, Chahed S, Sahin E, Dallery Y (2012) Modelling home care organisations from an operations management perspective. Flex Serv Manuf 26(3):295-319. doi:10.1007/s10696-012-9157-0

Piecyk M, Cullinane S, Edwards J (2012) Assessing the external impacts of freight transport. In: McKinnon P, Browne P, Whiteing A (eds) Green logistics: improving the environmental sustainability of logistics, 2nd edn. Kogan Page, London, pp 31-50

Rasmussen MS, Justesen T, Dohn A, Larsen J (2012) The home care crew scheduling problem: preference-based visit clustering and temporal dependencies. Eur J Oper Res 219(3):598-610. doi:10.1016/j.ejor.2011.10.048

Rest KD, Hirsch P (2016) Daily scheduling of home health care services using time-dependent public transport. Flex Serv Manuf 28(3):495-525. doi:10.1007/s10696-015-9227-1

Rest KD, Trautsamwieser A, Hirsch P (2012) Trends and risks in home health care. J Humanit Logist Supply Chain Manag 2(1):34-53. doi:10.1108/20426741211225993

Rosenfeld P, Russell D (2012) A review of factors influencing utilization of home and community-based long-term care: trends and implications to the nursing workforce. Policy Polit Nurs Pract 13(2):72-80. doi:10.1177/1527154412449747

Savelsbergh M (1992) The vehicle routing problem with time windows: minimizing route duration. ORSA J Comput 4(2):146-154. doi:10.1287/ijoc.4.2.146

Shoup D (1997) The high cost of free parking. J Plan Educ Res 17:3-20

Solomon MM (1987) Algorithms for the vehicle routing and scheduling problems with time window constraints. Oper Res 35(2):254-265. doi:10.1287/opre.35.2.254

Teal RF (1987) Carpooling: who, how and why. Transp Res A Gen 21(3):203-214. doi:10.1016/01912607(87)90014-8

Trautsamwieser A, Gronalt M, Hirsch P (2011) Securing home health care in times of natural disasters. OR Spectr 33(3):787-813. doi:10.1007/s00291-011-0253-4

UN General Assembly (2005) 2005 world summit outcome. In: A/60/L.1. 60th session of the general assembly, 30 Sept 2005, UN, New York

US-DOT (2013) 2013 DOT strategic sustainability performance plan. United States-Department of Transportation, Washington

Vanoutrive T, Vijver EVD, Malderen LV, Jourquin B, Thomas I, Verhetsel A, Witlox F (2012) What determines carpooling to workplaces in Belgium: location, organisation, or promotion? J Transp Geogr 22:77-86. doi:10.1016/j.jtrangeo.2011.11.006

Wartick SL (1980) Employer-organized vanpooling: a program for the 1980s. Bus Horiz 23(6):48-56. doi:10.1016/0007-6813(80)90050-6

Yalçındağ S, Matta A, Şahin E, Shanthikumar J (2016) The patient assignment problem in home health care: using a data-driven method to estimate the travel times of care givers. Flex Serv Manuf 28:1. doi:10.1007/s10696-015-9222-6

Christian Fikar is a post-doctoral researcher at the Institute for Production and Logistics at University of Natural Resources and Life Sciences, Vienna (BOKU). His research fields include logistics, supply chain management and related decision support systems to investigate and optimize delivery processes, particularly for humanitarian logistics and e-grocery operations.

Patrick Hirsch is an Assistant Professor, Project Manager and Deputy Head of PWL. He acts as a reviewer for several international scientific journals and is area Editor of the Journal of Applied Operational Research. His research interests are within transportation logistics, health care logistics and disaster management. He presented his work at several international conferences and published numerous book chapters as well as scientific journal articles. 